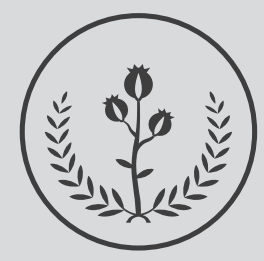

FUCS

\title{
Re

\section{Tuberculosis peritoneal y pleural en un paciente inmunocompetente con dolor abdominal crónico}

\section{Peritoneal and pleural tuberculosis in an immunocompetent patient with chronic abdominal pain}

Juan Sebastián Parra MD Juan Manuel Torres MD ${ }^{b}$ Diego Alejandro Ramírez MD $^{\mathrm{c}}$ Juan David Areiza MD Cristhian Felipe Ramírez MD $^{\mathrm{d}}$

\footnotetext{
a Semillero de investigación en Medicina Interna -SIMI. Médico residente de Cirugía General Universidad Militar Nueva Granada. Grupo de investigación MI-Dneuropsy, Facultad de Salud, Universidad Surcolombiana Neiva, Colombia.

${ }^{b}$ Cirugía General, Universidad Militar Nueva Granada, Bogotá, Colombia.

c Cirugia General Universidad Surcolombiana, Hospital Universitario Hernando Moncaleano Perdomo Neiva, Colombia.

${ }^{D}$ Medicina Interna. Instituto del Corazón y Metrosalud E.S.E. Cardiología. Universidad Pontificia Bolivariana, Medellín Colombia.
}

\section{R ES U M EN}

Objetivo: La tuberculosis es un grave problema de salud pública más acentuado en los países en desarrollo. De las manifestaciones extrapulmonares las que comprometen la cavidad abdominal están dentro de las menos frecuentes y se asocian con factores de predisposición específica. Se requiere un alto nivel de sospecha diagnóstica en el abordaje inicial de esta enfermedad. Presentamos el caso de un adulto masculino sin condiciones de predisposición quien consultó por un cuadro de dolor abdominal crónico y los estudios complementarios manifestaron una tuberculosis peritoneal con compromiso pleural sin síntomas respiratorios.

Palabras clave: tuberculosis peritoneal, laparoscopia, dolor abdominal, tuberculosis pleural

(C) 2021 Fundación Universitaria de Ciencias de la Salud -FUCS. Este es un artículo Open Access bajo la licencia CC BY-NC-ND (http:// creativecommons.org/licenses/by-nc-nd/4.0/)

\section{INFORMACIÓN DEL ARTÍCULO}

Historia del articulo: Fecha recibido: julio 19 de 2019 Fecha aceptado: julio 17 de 2020
Autor para correspondencia. Dr. Cristhian Felipe Ramírez: cristhianramos1989@hotmail.com
DOI

10.31260/RepertMedCir.01217372.946 
Objetive: Tuberculosis is a serious public health problem most prevalent in developing countries. Of extrapulmonary manifestations, those involving the abdominal cavity are among the least frequent and are associated with specific predisposing factors. A high level of diagnostic suspicion is required in the initial approach of this disease. We present the case of a male adult patient with no predisposing conditions who consulted for chronic abdominal pain. The complementary studies evidenced peritoneal tuberculosis with pleural involvement with no respiratory symptoms.

Keywords: peritoneal tuberculosis, laparoscopy, abdominal pain, pleural tuberculosis.

(C) 2021 Fundación Universitaria de Ciencias de la Salud - FUCS. This is an open access article under the CC BY-NC-ND license (http://creativecommons.org/licenses/by-nc-nd/4.0/).

INTRODUCCIÓN

La tuberculosis continúa siendo un grave problema de salud pública, presentándose de manera frecuente en países de bajos ingresos y climas tropicales. Globalmente el $23 \%$ de la población mundial se estimó que estaba infectada con Mycobacterium tuberculosis complex en 2014, lo que equivale a 1.7 billones de personas. ${ }^{1}$ Para 2016 se calcularon alrededor de 10.4 millones de casos incidentes, 1 millón de estos en personas con infección por el virus de inmunodeficiencia humana (VIH). ${ }^{2} \mathrm{Si}$ bien la mortalidad cayó de 1.7 millones en el año 2000 a 1.3 millones en 2016, está condición sigue considerándose la principal causa de muerte por enfermedad infecciosa en el mundo y en la historia de la humanidad. ${ }^{2}$ El $82 \%$ de las muertes por tuberculosis en personas sin VIH ocurrieron en Africa $(32 \%)$ y el sudeste Asiático $(50 \%){ }^{2}$ El incremento de personas infectadas por VIH y de quienes requieren medicamentos inmunosupresores ha aumentado el número de casos en el mundo occidental. ${ }^{3}$ El compromiso pulmonar es el más común. ${ }^{4}$ De las formas extrapulmonares que corresponden al $20 \%$ de todos los $\operatorname{casos}^{5}$, la presentación abdominal es la número seis en frecuencia $(10 \%$ de las formas extrapulmonares $)^{6}$ y puede comprometer estructuras luminales (intestino, peritoneo, ganglios linfáticos) y órganos viscerales (páncreas o hígado). ${ }^{7}$ La variedad de presentación depende de la cantidad de líquido o reacción fibrótica; puede ocurrir en una forma húmeda asociada con ascitis o resultar en lesiones fijas en las cuales predominan las adherencias y la fibrosis. ${ }^{8}$ Hay 4 grupos de presentación: linfadenopatia, peritoneal, gastrointestinal y visceral. ${ }^{9} \mathrm{La}$ zona ileocecal y la linfadenopatía abdominal son el área y grupo de presentación más común. ${ }^{10}$

Para sufrir esta forma de la enfermedad hay factores de riesgo establecidos como infección por VIH, cirrosis, diálisis peritoneal o trasplante de organo con inmunosupresión; sin embargo, para identificarla siempre se debe tener una alta sospecha diagnóstica. ${ }^{11}$ Presentamos el caso de un paciente masculino en la quinta década de la vida sin factores de riesgo para tuberculosis abdominal, con un cuadro de síntomas predominantes en esta localización (dolor abdominal), quien luego de los estudios diagnósticos se comprobó que cursaba con una tuberculosis peritoneal y pleural, sin compromiso parenquimatoso pulmonar.

\section{DESCRIPCIÓN DEL CASO}

Paciente de 41 años, masculino, sin antecedentes patológicos de importancia, procedente de un centro penitenciario local, con cuadro clínico de 6 meses de evolución de dolor abdominal generalizado con predomino en epigástrico de tipo urente y en hipogastrio con cólico, no referido ni irradiado, sin asociarse con alimentación ni cambios del hábito intestinal, como tampoco a otros síntomas abdominales; el dolor se presentaba en episodios de minutos a varias horas durante el día, pero en los 2 meses antes del ingreso la aparición fue diaria. En la revisión por sistemas no había episodios de fiebre, pero como hallazgo positivo refirió pérdida de peso involuntaria de cerca de 12 kilogramos en los últimos 6 meses. Al examen físico de ingreso los signos vitales eran normales, a nivel abdominal aparecía dolor a la palpación profunda, pero sin masas ni signos de irritación peritoneal y el resto del examen físico sin alteraciones. Los datos paraclínicos de ingreso (tabla 1) evidenciaron reactante de fase aguda positivo, neutrofilia sin leucocitosis, anemia leve, trombocitosis, prueba de virus de inmunodeficiencia humana (VIH) negativa, sin más alteraciones de importancia. En las imágenes había evidencia de derrame pleural derecho sin otras anormalidades pulmonares a nivel del parenquima pulmonar, por lo que no se consideró realizar tomografía computarizada (figura 1). Se procedió a realizar estudio de líquido pleural previa verificación de conteo de plaquetas y tiempo de coagulación en rango de seguridad; los resultados del estudio citoquimico fueron compatibles con un exudado neutrofílico, adenosin deaminasa debajo del punto de corte significativo para tuberculosis pleural y tinciones para microorganismos negativas (baciloscopia). 
- Repertorio.

Tabla 1. Numero de artículo por términos mesh

\begin{tabular}{|c|c|c|}
\hline Laboratorio & Resultado & Valor de referencia \\
\hline Bilirrubina total & $0.39 \mathrm{mg} / \mathrm{dL}$ & $0.2-1$ \\
\hline Bilirrubina directa & $0.16 \mathrm{mg} / \mathrm{dL}$ & \\
\hline Glicemia & $105.92 \mathrm{mg} / \mathrm{dL}$ & $74-106$ \\
\hline Nitrógeno ureico & $7.1 \mathrm{mg} / \mathrm{dL}$ & $7-18$ \\
\hline Creatinina & $0.85 \mathrm{mg} / \mathrm{dL}$ & $0.67-1.17$ \\
\hline Transaminasa oxalacética & $32 \mathrm{UI} / \mathrm{L}$ & $15-37$ \\
\hline Transaminasa pirúvica & $20 \mathrm{UI} / \mathrm{L}$ & $16-63$ \\
\hline Amilasa & $26.64 \mathrm{U} / \mathrm{L}$ & $25-115$ \\
\hline Proteína $\mathrm{C}$ reactiva & $18.16 \mathrm{mg} / \mathrm{dL}$ & $0-6$ \\
\hline Leucocitos & $8.500 \mathrm{~mm} 3$ & $4.800-10.800$ \\
\hline Neutrófilos & $7.000 \mathrm{~mm} 3$ & \\
\hline Linfocitos & 900 mm3 & \\
\hline Hemoglobina & $10.3 \mathrm{~g} / \mathrm{dL}$ & $14-18$ \\
\hline Hematocrito & $32.2 \%$ & $42-52$ \\
\hline Volumen corpuscular medio & $77.1 \mathrm{fL}$ & $80-100$ \\
\hline Hemoglobina corpuscular media & $24.6 \mathrm{pg}$ & $27-31$ \\
\hline Plaquetas & 734.000 & $150.000-450.000$ \\
\hline VIH & Negativo & \\
\hline Hormona estimulante de la tiroides (TSH) & $2.28 \mathrm{uUI} / \mathrm{ml}$ & $0.4-4$ \\
\hline Determinación cualitativa de anticuerpos contra Treponema pallidum & Negativo & \\
\hline Citoquímico de líquido pleural & $\begin{array}{l}\text { Color: amarillo pálido después de centrifugar } \\
\text { Aspecto: ligueramente turbio } \\
\text { Coagulación: positiva } \\
\text { Recuento de leucocitos: } 12 \mathrm{~mm} 3 \\
\text { Neutrófilos: } 70 \% \\
\text { Linfocitos: } 30 \% \\
\text { Recuento de hematíes: } 3600 \mathrm{~mm} 3 \\
\text { Hematíes frescos: } 60 \% \\
\text { Hematíes crenados: } 40 \% \\
\text { Glucosa: } 10.7 \mathrm{mg} / \mathrm{dL} \\
\text { Proteínas: } 6.49 \mathrm{~g} / \mathrm{dL} \\
\text { Colesterol: } 76.5 \mathrm{mg} / \mathrm{dL} \\
\text { Amilasa: } 16.7 \mathrm{UI} / \mathrm{L} \\
\text { LDH: } 1501 \mathrm{UI} \\
\text { ADA: } 12 \mathrm{U} / \mathrm{L} \\
\text { Baciloscopia: negativa. }\end{array}$ & \\
\hline LDH & $226.8 \mathrm{U} / \mathrm{L}$ & $85-227$ \\
\hline Proteínas totales & $7.81 \mathrm{~g} / \mathrm{dL}$ & $6.4-8.2$ \\
\hline \multicolumn{3}{|l|}{ Tiempos de coagulación. } \\
\hline TP & 13.2 seg control 13.5 INR 0.98 & $11.7-15.3 \mathrm{seg}$ \\
\hline TPTa & 34.9 seg control 31.4 seg & 23.6-34.8 seg \\
\hline Cultivo de líquido pleural y peritoneal. & Positivo en medio líquido para Mycobacterium tuberculosis. & \\
\hline
\end{tabular}

Fuente: Ios autores. 


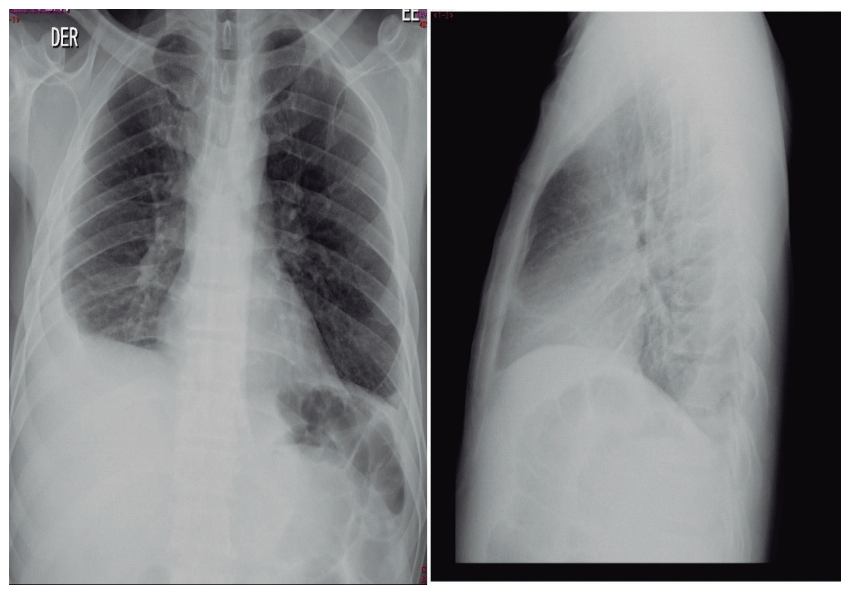

Figura 1. Radiografía de tórax, proyección PA y lateral. Se evidencia derrame pleural derecho sin cambios parenquimatosos pulmonares de importancia que sugieran enfermedad pulmonar activa o infección previa por tuberculosis. Fuente: los autores.

De manera inicial y descartando una infección por VIH ante la edad del paciente, se procedió con la toma de imágenes en busca de patología de neoplasia. Con los resultados del líquido pleural realizado de manera inicial (exudado neutrofilico, ADA negativo) no se consideró la posibilidad de una tuberculosis como causa de la condición clínica del paciente. Durante la hospitalización y en espera de los estudios diagnósticos de extensión el paciente presentó agudización del dolor abdominal que era ya de predominio epigástrico, haciéndose difuso a la palpación sin signos de irritación peritoneal, se tornó febril y taquicárdico con empeoramiento del patrón respiratorio. La tomografía abdominal total reveló un leve aumento en la densidad mesentérica con formaciones nodulares pequeñas de hasta $9 \mathrm{~mm}$ de diámetro (figura 2). A las pocas horas de realizado dicho estudio desarrolló signos de irritación peritoneal y se decidió una laparoscopia diagnóstica donde se notó una gran cantidad de lesiones pequeñas de tamaño variable de color blanquecino que tapizaban la pared peritoneal y las asas intestinales, con líquido libre de color cetrino. El resultado de la biopsia de peritoneo mostró presencia de múltiples granulomas epitelioides acompañados de células gigantes multinucleadas con centro necrótico y escasos linfocitos en la periferia. La tinción para bacilos ácido alcohol resistentes fue negativa en dicho material histopatológico y el reporte del cultivo del líquido peritoneal y pleural mostró posteriormente crecimiento de Mycobacterium tuberculosis complex. Bajo la consideración de una tuberculosis pulmonar asociada se decidió llevar a broncoscopia diagnóstica para toma de muestras, con resultados de tinciones directas negativas (baciloscopia, KOH) y estudios de microbiología (cultivo para tuberculosis en medio sólido y líquido) sin anormalidades. No se realizaron estudios de biología molecular (Xpert-Gene) por no disponerse. El paciente fue dado de alta con manejo de primera fase para tuberculosis en las semanas siguientes de lograr la estabilidad clínica.

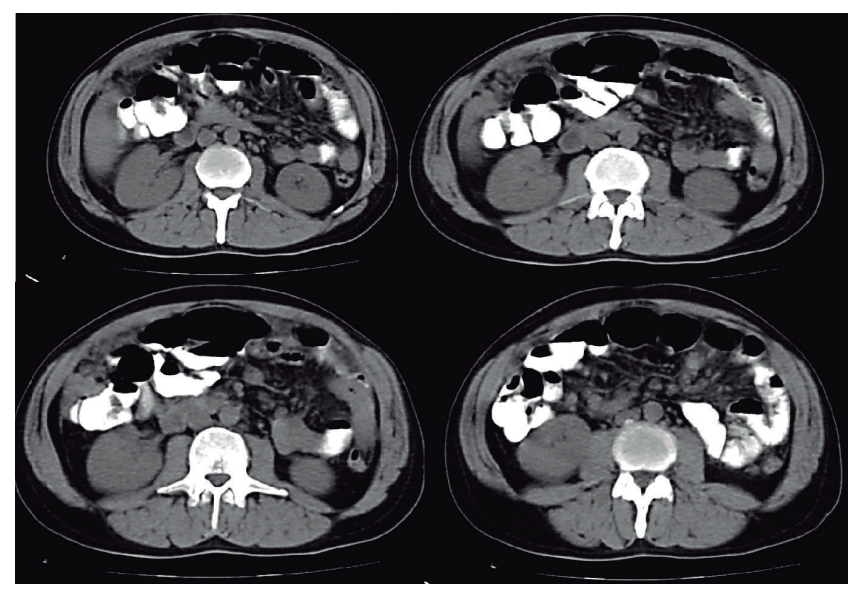

Figura 2. Tomografía de abdominal total. Los cortes axiales en ventana abdominal con contraste oral muestran leve aumento en la densidad mesentérica con formaciones nodulares pequeñas de hasta $9 \mathrm{~mm}$ de diámetro. Fuente: los autores.

\section{DISCUSIÓN}

Presentamos el caso de un paciente con tuberculosis peritoneal con compromiso concomitante pleural y sin evidencia de afectación pulmonar. La tuberculosis abdominal es común en los países en vía de desarrollo. ${ }^{12}$ En localizaciones de baja prevalencia de tuberculosis se asocia con condiciones de predisposición como infección por VIH/ SIDA, inmigrantes de áreas endémicas de tuberculosis, pobreza en zonas urbanas y edad avanzada. ${ }^{13}$

A pesar de la tendencia actual en cuanto a las tasas de incidencia de la enfermedad que de manera global han mostrado una leve disminución progresiva, los casos de compromiso extrapulmonar han aumentado de $16 \%$ en 1993 a $20 \%$ en el año $2008 .{ }^{11}$ La tuberculosis peritoneal es la principal presentación del compromiso intrabdominal y representa cerca de $6,1 \%$ de todos los casos de la forma extrapulmonar. ${ }^{7}$ Es predominante en personas entre la tercera y cuarta década de la vida, pero puede ocurrir a cualquier edad. ${ }^{8}$ Las mujeres se afectan más en países en vía de desarrollo ${ }^{14}$, algo no notado en las naciones del primer mundo. ${ }^{15}$ El desarrollo de la tuberculosis peritoneal se ha asociado con varios factores de riesgo como infección por VIH, enfermedad hepática alcohólica, ser nativo americano, enfermedad renal crónica en diálisis peritoneal, diabetes mellitus, cáncer y uso de corticoesteroides y medicamentos inmunosupresores. ${ }^{11}$ Hay varias formas por las cuales la tuberculosis puede comprometer el abdomen9: primero el bacilo puede entrar al tracto intestinal a través de la ingestión de leche o esputo, la capa mucosa puede infectarse con bacilos formando tuberculos epitelioides en el tejido linfático de la submucosa. Después de 2 a 4 semanas, la necrosis caseosa de los tuberculos conduce a ulceración de la mucosa con extensión a capas más profundas y tejido linfoide adyacente, así como en el 


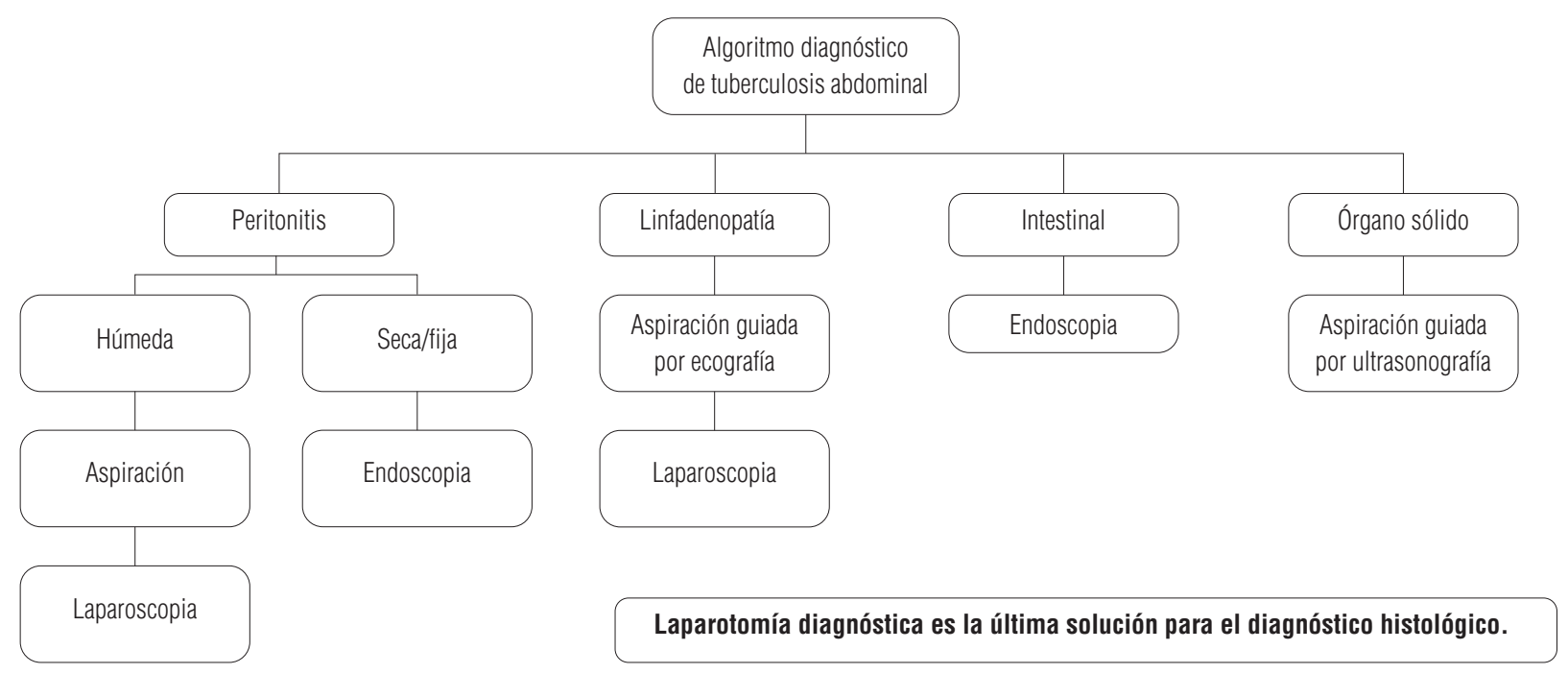

Figura 3. Algoritmo diagnóstico en tuberculosis abdominal. Tomado de Abu-Zidan y col. ${ }^{19}$

peritoneo. Rara vez estos bacilos entran en la circulación portal o arterial hepática para comprometer organos como el hígado, páncreas o bazo. ${ }^{9}$ La segunda manera es vía extensión hematógena de focos en otras localizaciones como órganos sólidos abdominales, riñón, ganglios linfáticos y peritoneo. ${ }^{9}$ La tercera implica infección directa desde focos adyacentes como trompas de Falopio y anexos, abscesos del psoas y espondilitis. ${ }^{9}$ Finalmente también se puede diseminar por canales linfáticos de ganglios infectados. ${ }^{9}$ Es frecuente el compromiso extraabdominal ${ }^{16}$ y 15 a $20 \%$ de estos pacientes tienen enfermedad pulmonar activa. El Mycobacterium tuberculosis es el agente más importante y en algunos escenarios el $\mathrm{M}$. bovis cuando hay consumo de leche no pasteurizada. ${ }^{17}$ Nuestro paciente no tenía ningún factor de riesgo para tuberculosis peritoneal, sin embargo la condición de recluso favorece el desarrollo de tuberculosis. Cabe anotar que tenía compromiso pleural sin evidencia radiográfica o microbiológica de lesión pulmonar activa.

La enfermedad tiene un curso insidioso con fase subaguda de difícil diagnóstico si no se sospecha. El promedio de duración de los síntomas es de varias semanas a meses y los dos más frecuentes son dolor abdominal (31 a 94\%) y fiebre (45 a 100\%). Las manifestaciones sistémicas son más prominentes en la tuberculosis peritoneal que en otras formas. La diarrea es inusual y una minoría puede tener síntomas respiratorios. Los hallazgos del examen físico más comunes son ascitis $(73 \%$ ) y sensibilidad abdominal ( $47.7 \%)$. La ausencia de signos de enfermedad hepática crónica en contexto de ascitis debe elevar la sospecha diagnostica8, $50 \%$ de los pacientes tiene prueba de tuberculina positiva sin conocer la utilidad en esta forma de presentación de la enfermedad. Los otros hallazgos paraclínicos importantes son anemia normocítica normocrómica y VSG elevada ${ }^{8} ; 19$ al $83 \%$ presentan anormalidades en la radiografía de tórax ya sea por tuberculosis activa o cicatricial $(33 \%) \cdot{ }^{18}$ Los estudios del líquido ascítico muestran un exudado linfocitico con un gradiente de albumina menor de 1.1 y un nivel de proteínas mayor de $2.5 \mathrm{~g} / \mathrm{dL}$, con conteos de células que oscilan alrededor de 500 a $1500 / \mathrm{mm} 3$ de predominio linfocitico (40$92 \%) .{ }^{8}$ Como se nota, los hallazgos clínicos y radiológicos de la tuberculosis abdominal no son patognomónicos. Debido al tiempo requerido para el crecimiento del microorganismo en el cultivo, se debe intentar hacer un diagnóstico histopatológico temprano para iniciar el diagnóstico. AbuZidan y col. sugieren un algoritmo diagnóstico ${ }^{19}$ interesante dependiendo de la categorización de la enfermedad en 5 grupos (figura 3): abdominal, lesión de órgano sólido, linfadenopatía, peritonitis húmeda y peritonitis fija o seca. El diagnóstico de la tuberculosis gastrointestinal puede lograrse con endoscopia y biopsia y su rendimiento se incrementa con el aumento del número de biopsias. Se reporte que las tomadas por colonoscopia en 50 pacientes con tuberculosis colónica fueron diagnósticas en 40 de estos $(80 \%) .{ }^{20}$ Los hallazgos de endoscopia se han reportado como lesiones con apariencia de ulcera en $60 \%$, ulcerohipertróficas $30 \%$ e hipertróficas en $10 \% \cdot{ }^{10}$ Otras investigaciones han informado úlceras $(70 \%)$, nódulos $(56 \%)$, válvula ileocecal deformada $(40 \%)$, constricciones $(23 \%)$, pólipos $(14 \%)$ y bandas fibróticas $(7 \%) .{ }^{21}$

El diagnóstico de lesiones de órganos sólidos se puede lograr con biopsia guiada por ultrasonido. La peritonitis húmeda y la linfadenopatía pueden diagnosticarse por medio de la aspiración guiada por ultrasonido, seguida por una laparoscopia si es necesario. La laparotomía diagnóstica debe ser la última opción para lograr el diagnóstico histológico. No se incluye aquí la enteroscopia y la capsula endoscopica debido a los datos limitados de estas modalidades.

En tuberculosis peritoneal el diagnóstico se realiza con confirmación microbiológica o patológica teniendo como estándar de oro la biopsia peritoneal y la laparoscopia. ${ }^{22}$ 
Tabla 2. Diagnóstico diferencial de tuberculosis abdominal

\begin{tabular}{|l|l|}
\hline Tuberculosis peritoneal & Tuberculosis intestinal \\
\hline Carcinomatosis & Enfermedad de Crohn \\
Peritonitis bacteriana & Linfoma intestinal \\
Peritonitis por talco & Carcinoma \\
\hline Enfermedad hepática crónica & Infecciones por yersinia \\
& Ameboma \\
\hline
\end{tabular}

Fuente: los autores.

Los hallazgos de ultrasonografía incluyen ascitis (30 a $100 \%$ ), detritus ecogénicos y/o engrosamiento peritoneal..$^{23}$ Latomografía esmás sensible para evidenciar elengrosamiento y las adenopatías, teniendo en cuenta que la combinación de macronódulos mesentéricos, engrosamientos lisos del peritoneo, masas nodales con centros hipodensos, lesiones esplénicas y calcificaciones ayudan a realizar la distinción con carcinomatosis peritoneal ${ }^{24}$; algunos de los cuales fueron notados en el paciente. Estos estudios de imágenes permiten también realizar toma de muestras de los nódulos para pruebas microbiológicas. Las tinciones y cultivos de líquido ascítico tienen baja sensibilidad, reportada entre 3 y $35 \%$, mientras la del cultivo puede incrementarse hasta $83 \% .^{17}$ La sensibilidad y especificidad reportada de la adenosin deaminasa es $100 \%$ y $97 \%$ con un punto de corte de 36 a $40 \mathrm{UI} / \mathrm{L} \cdot{ }^{25} \mathrm{La}$ laparoscopia permite la inspección peritoneal y la toma de muestras para estudios microbiológicos y de histopatología, con lo cual se logra confirmar en $85-90 \%$ de los casos. La apariencia ha sido clasificada en 3 tipos, peritoneo engrosado con nódulos miliares blanquecinos y ascitis $(66 \%)$, engrosamiento peritoneal con adherencias y ascitis $(21 \%)$ y el tipo fibroaditivo donde el peritoneo está muy engrosado con nódulos amarillos y material similar al queso con adherencias extensas $(13 \%) .{ }^{26}$ La sensibilidad y especificidad para el diagnóstico han sido reportados entre $93 \%$ y $98 \%{ }^{8}$

El tratamiento es médico y se hace de manera similar a la tuberculosis pulmonar ${ }^{11}$, con un papel controversial en cuanto a la utilización de corticoides por los escasos estudios reportados, pero parecen tener una acción positiva en los pacientes con tuberculosis peritoneal. ${ }^{27}$ El retraso en el tratamiento puede aumentar la morbimortalidad con deterioro clínico en $80 \%$ de los pacientes y mortalidad asociada que llega hasta $34 \% .{ }^{17}$ La intervención quirúrgica se reserva para los pacientes que presenten complicaciones por la inflamación y las adherencias como perforación, obstrucción, fístulas, abscesos y hemorragia. ${ }^{11}$ Hay que tener presente que entre 20 y $40 \%$ de los pacientes con tuberculosis abdominal presentan abdomen agudo y necesidad de manejo quirúrgico. ${ }^{28}$ En los casos crónicos con obstrucción subaguda el manejo debe ser conservador y la cirugía debe planearse después, según la necesidad de la misma. ${ }^{28}$ Como es una enfermedad sistémica la resección quirúrgica debe minimizarse, las constricciones del intestino delgado se tratan con liberación de las mismas y evitar mayores resecciones. ${ }^{28}$ Una alternativa es la dilatación con balón por medio de endoscopia dependiendo de la accesibilidad de las mismas; aun con experiencia limitada esta técnica parece ser segura y puede evitar la necesidad de un procedimiento mayor. ${ }^{28}$ La peritonitis aguda y la linfadenitis mesentérica deben manejarse con precaución. Si la laparotomía es necesaria, se limitará a la toma de las biopsias necesarias con lavado peritoneal y cierre sin drenajes. ${ }^{28}$ Debido a la forma de presentación de esta enfermedad y los hallazgos de laboratorio poco específicos, se ha considerado una gran imitadora. El principal diagnóstico diferencial es la enfermedad de Crohn teniendo presente que es una entidad infrecuente, afecta ciertos grupos poblaciones como caucásicos y con un pico entre los 20-40 años. Aunque la enfermedad perianal y las fistulas entéricas pueden ser de etiología tuberculosa, estas son menos comunes si se compara con la enfermedad de Crohn. En la tabla 2 se muestran los principales diagnósticos diferenciales. Las personas con enfermedad hepática de base, infección por VIH o malignidad, tienen mayor mortalidad y peor pronóstico. ${ }^{8}$

\section{CONCLUSIÓN}

La tuberculosis es un problema global de salud. La presentación abdominal está dentro de las menos frecuentes siendo los compromisos ileocecal y peritoneal los más reportados. Es un reto diagnóstico por la forma de presentación y puede imitar otra enfermedad. El retraso en el diagnóstico se relaciona con aumento de las complicaciones y la mortalidad. En su valoración diagnóstica son útiles los estudios imageneológicos y endoscópicos. La laparoscopia debe considerarse en etapa temprana para obtener muestras para estudios histológicos pues el pronto diagnóstico y tratamiento reducen la morbilidad y mortalidad.

CONFLICTOS DE INTERÉS

Los autores manifiestan no tener conflictos de interés 
REFEREN CIAS

1. Houben RMGJ, Dodd PJ. The Global Burden of Latent Tuberculosis Infection: A Re-estimation Using Mathematical Modelling. PLoS Med. 2016;13(10):e1002152. doi: 10.1371/journal.pmed.1002152

2. Glaziou P, Floyd K, Raviglione MC. Global Epidemiology of Tuberculosis. Semin Respir Crit Care Med. 2018;39(3):271-85. doi: 10.1055/s-0038-1651492

3. Sharma V, Singh H, Mandavdhare HS. Tubercular Abdominal Cocoon: Systematic Review of an Uncommon Form of Tuberculosis. Surg Infect. 2017;18(6):736-41. doi: 10.1089/sur.2017.110

4. Lyon SM, Rossman MD. Pulmonary Tuberculosis. Microbiol Spectr. 2017;5(1). doi: 10.1128/microbiolspec.TNMI7-0032-2016

5. Yang Z, Kong Y, Wilson F, Foxman B, Fowler AH, Marrs CF, et al. Identification of risk factors for extrapulmonary tuberculosis. Clin Infect Dis. 2004;38(2):199-205. doi: 10.1086/380644

6. Rathi P, Gambhire P. Abdominal Tuberculosis. J Assoc Physicians India. 2016;64(2):38-47.

7. Sharma MP, Bhatia V. Abdominal tuberculosis. Indian J Med Res. 2004;120(4):305-15.

8. Sanai FM, Bzeizi KI. Systematic review: tuberculous peritonitis-presenting features, diagnostic strategies and treatment. Aliment Pharmacol Ther. 2005;22(8):685-700. doi: 10.1111/j.13652036.2005.02645.x

9. Debi U, Ravisankar V, Prasad KK, Sinha SK, Sharma AK. Abdominal tuberculosis of the gastrointestinal tract: Revisited. World J Gastroenterol WJG. 2014;20(40):14831-40. doi: 10.3748/wjg.v20. i40.14831

10. Lazarus AA, Thilagar B. Abdominal tuberculosis. Dis--Mon DM. 2007;53(1):32-8. doi: 10.1016/j.disamonth.2006.10.004

11. Vaid U, Kane GC. Tuberculous Peritonitis. Microbiol Spectr. 2017;5(1). doi: 10.1128/microbiolspec.TNMI7-0006-2016

12. Ihekwaba FN. Abdominal tuberculosis: a study of 881 cases. J R Coll Surg Edinb. 1993;38(5):293-5.

13. Rieder HL, Cauthen GM, Kelly GD, Bloch AB, Snider DE. Tuberculosis in the United States. JAMA. 1989;262(3):385-9.

14. Borhanmanesh F, Hekmat K, Vaezzadeh K, Rezai HR. Tuberculous peritonitis. Prospective study of 32 cases in Iran. Ann Intern Med. 1972;76(4):567-72. doi: 10.7326/0003-4819-76-4-567

15. Tan K-K, Chen K, Sim R. The spectrum of abdominal tuberculosis in a developed country: a single institution's experience over 7 years. J Gastrointest Surg Off J Soc Surg Aliment Tract. 2009;13(1):142-7. doi: 10.1007/s11605-0080669-6

16. Reto Valiente L, Pichilingue Reto C, Pichilingue Prieto O, Dolores Cerna K. Tuberculosis abdominal en niños y adolescentes. Un desafío diagnóstico. Rev Gastroenterol Perú. 2015;35(4):318-22.

17. Veeragandham RS, Lynch FP, Canty TG, Collins DL, Danker WM. Abdominal tuberculosis in children: review of 26 cases. J Pediatr Surg. 1996 Jan;31(1):170-5; discussion 175-176. doi: 10.1016/S0022-3468(96)90342-5
18. Chow KM, Chow VCY, Hung LCT, Wong SM, Szeto CC. Tuberculous peritonitis-associated mortality is high among patients waiting for the results of mycobacterial cultures of ascitic fluid samples. Clin Infect Dis Off Publ Infect Dis Soc Am. 2002;35(4):409-13. doi: $10.1086 / 341898$

19. Abu-Zidan FM, Sheek-Hussein M. Diagnosis of abdominal tuberculosis: lessons learned over 30 years: pectoral assay. World J Emerg Surg WJES. 2019;14:33. doi: 10.1186/s13017-019-0252-3

20. Shah S, Thomas V, Mathan M, Chacko A, Chandy G, Ramakrishna BS, et al. Colonoscopic study of 50 patients with colonic tuberculosis. Gut. 1992;33(3):347-51. doi: 10.1136/gut.33.3.347

21. Alvares JF, Devarbhavi H, Makhija P, Rao S, Kottoor R. Clinical, colonoscopic, and histological profile of colonic tuberculosis in a tertiary hospital. Endoscopy. 2005;37(4):351-6. doi: 10.1055/s2005-861116

22. Chow KM, Chow VC-Y, Szeto CC. Indication for peritoneal biopsy in tuberculous peritonitis. Am J Surg. 2003;185(6):567-73. doi: 10.1016/s0002-9610(03)00079-5

23. Sheikh M, Abu-Zidan F, al-Hilaly M, Behbehani A. Abdominal tuberculosis: comparison of sonography and computed tomography. J Clin Ultrasound JCU. 1995;23(7):413-7. doi: 10.1002/jcu.1870230704

24. Rodríguez E, Pombo F. Peritoneal tuberculosis versus peritoneal carcinomatosis: distinction based on CT findings. J Comput Assist Tomogr. 1996;20(2):269-72. doi: 10.1097/00004728-19960300000018

25. Riquelme A, Calvo M, Salech F, Valderrama S, Pattillo A, Arellano $M$, et al. Value of adenosine deaminase (ADA) in ascitic fluid for the diagnosis of tuberculous peritonitis: a meta-analysis. J Clin Gastroenterol. 2006;40(8):705-10. doi: 10.1097/00004836200609000-00009

26. Bhargava DK, Shriniwas null, Chopra P, Nijhawan S, Dasarathy S, Kushwaha AK. Peritoneal tuberculosis: laparoscopic patterns and its diagnostic accuracy. Am J Gastroenterol. 1992;87(1):109-12.

27. Soni H, Bellam BL, Rao RK, Kumar PM, Mandavdhare HS, Singh $\mathrm{H}$, et al. Use of steroids for abdominal tuberculosis: a systematic review and meta-analysis. Infection. 2019;47(3):387-94. doi: 10.1007/s15010-018-1235-0

28. Weledji EP, Pokam BT. Abdominal tuberculosis: Is there a role for surgery? World J Gastrointest Surg. 2017;9(8):174-81. doi: 10.4240/wjgs.v9.i8.174 